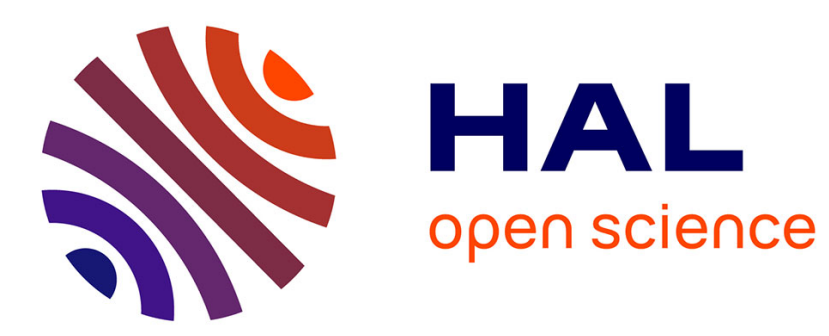

\title{
Fiber alignment in oscillating confined shearing flows
}

Scott Strednak, Jason E Butler, Laurence Bergougnoux, Elisabeth Guazzelli

\section{To cite this version:}

Scott Strednak, Jason E Butler, Laurence Bergougnoux, Elisabeth Guazzelli. Fiber alignment in oscillating confined shearing flows. Physical Review Fluids, 2021, 6, 10.1103/physrevfluids.6.014302 . hal-03117749

\section{HAL Id: hal-03117749 \\ https://hal.science/hal-03117749}

Submitted on 21 Jan 2021

HAL is a multi-disciplinary open access archive for the deposit and dissemination of scientific research documents, whether they are published or not. The documents may come from teaching and research institutions in France or abroad, or from public or private research centers.
L'archive ouverte pluridisciplinaire HAL, est destinée au dépôt et à la diffusion de documents scientifiques de niveau recherche, publiés ou non, émanant des établissements d'enseignement et de recherche français ou étrangers, des laboratoires publics ou privés. 


\title{
Fiber alignment in oscillating confined shearing flows
}

\author{
Scott Strednak $\odot,{ }^{1}$ Jason E. Butler $\odot,{ }^{1, *}$ Laurence Bergougnoux $\odot,{ }^{2}$ and Élisabeth Guazzelli $\odot^{3}$ \\ ${ }^{1}$ Department of Chemical Engineering, University of Florida, Gainesville, Florida 32611, USA \\ ${ }^{2}$ Aix-Marseille Université, CNRS, IUSTI, Marseille, France \\ ${ }^{3}$ Université de Paris, CNRS, Matière et Systèmes Complexes (MSC) UMR 7057, Paris, France
}

(Received 25 July 2020; accepted 14 December 2020; published 7 January 2021)

\begin{abstract}
Fiber orientation in oscillatory shearing flow was studied both experimentally and numerically. Optical measurements were made using a custom flow cell containing rigid, noncolloidal fibers suspended at high concentration in a Newtonian fluid. Simulations that account for hydrodynamic drag and excluded volume predict fiber alignment in the vorticity direction for some conditions, in agreement with the measurements. Vorticity alignment was found to be a complex function of strain amplitude and fiber concentration, confinement, and aspect ratio.
\end{abstract}

DOI: 10.1103/PhysRevFluids.6.014302

\section{INTRODUCTION}

Shearing concentrated particle suspensions using an oscillatory flow can create a microstructure that is highly organized and substantially different from that observed in steady shearing flows. For suspensions of hard spheres that are Brownian, techniques such as light scattering [1,2] and microscopy [3-5] have established the existence of multiple phases, including face-centered-cubic and string phases. The microstructure is controlled in part by the rate of shear, or frequency of oscillation, relative to the timescale for Brownian diffusion. However, the amplitude of the oscillatory strain largely controls the microstructural phase [2,5,6].

Highly concentrated suspensions of non-Brownian spheres also organize under oscillatory flow. In this case, the strain amplitude and particle concentration control the microstructure; the rate of shear (i.e., frequency of oscillation) is irrelevant so long as it is maintained within the creeping-flow regime and since there is no Brownian timescale. Evidence for the strain amplitude-dependent organization is given by rheological measurements $[7,8]$ which have been correlated to the microstructure through simulation [9]. Changes in the microstructure are driven, at least partially, by a transition from reversible dynamics at small strain amplitudes to irreversible dynamics at large strain amplitudes, where the transition depends on the concentration of particles $[10,11]$.

Oscillatory rheology is used commonly to characterize colloidal and noncolloidal particle suspensions for materials development and quality control, hence the interest in examining the relationship between the applied flow, microstructure, and rheology. The possibility of using oscillatory flows to alter favorably the microstructure for specific applications has also been suggested [2]. For example, investigations have explored using oscillatory shear to assemble colloidal crystals $[12,13]$, and oscillatory shear may be relevant for self-assembly of noncolloidal particles as well [14].

The vast majority of studies concerning oscillatory flow of concentrated particle suspensions have been limited to spheres rather than other particle shapes, which comprise many suspensions. In the case of elongated particles that are non-Brownian and suspended at high concentration, oscillating the flow can induce strong alignment in the vorticity direction [15,16]. This alignment contrasts with the tendency of fibers to align with flow direction when sheared steadily within

\footnotetext{
*Corresponding author: butler@ che.ufl.edu
} 

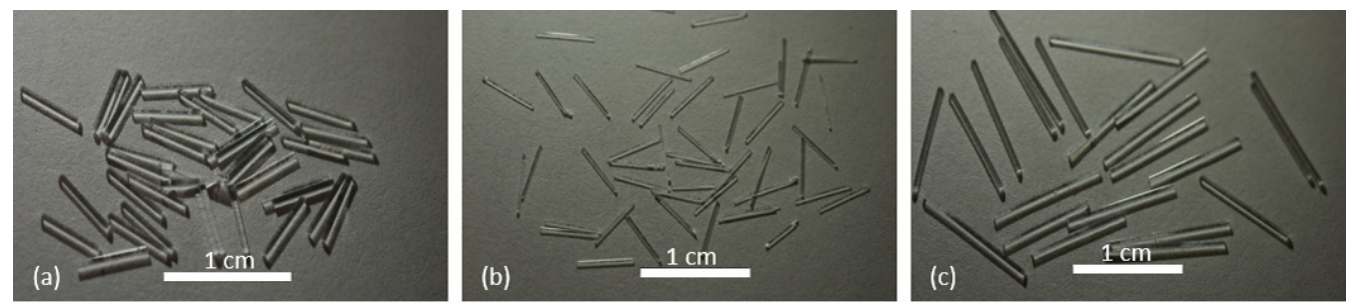

FIG. 1. Fibers were manufactured from the poly(methyl methacrylate) core of fiber optic cables of diameter $d$ by cutting them in segments of length $L$. Various aspect ratios of $A=L / d$ were generated: (a) $L=5.2 \pm$ $0.2 \mathrm{~mm}, d=0.46 \pm 0.06 \mathrm{~mm}$, and $A=11 \pm 2$; (b) $L=5.2 \pm 0.2 \mathrm{~mm}, d=0.23 \pm 0.02 \mathrm{~mm}$, and $A=23 \pm 2$; and (c) $L=10.4 \pm 0.2 \mathrm{~mm}, d=0.46 \pm 0.06 \mathrm{~mm}$, and $A=23 \pm 2$.

a Newtonian fluid. Simulations by Snook et al. [17] accurately predicted the alignment of the fibers and indicated that particle collisions drive the organization of the orientation distribution. Additionally, they speculated that the suspension must be confined in order for vorticity alignment to occur.

Here, we address questions raised by the work of Franceschini et al. $[15,16]$ and Snook et al. [17], including the effects of fiber concentration and confinement on the orientation distribution. Measurements of fiber alignment were made in a custom-built flow cell as functions of fiber geometry and strain amplitude, as well as concentration and confinement (see Sec. II). Simulation methods used to predict the orientation are described in Sec. III. Section IV B demonstrates that the measurements are consistent with previous experiments at the same conditions, though the dependence of alignment on the distance from the bounding walls must be considered when making the comparisons (see Sec. IV A). Additional results in Sec. IV confirm that vorticity alignment depends upon the confinement and concentration of particles, as well as particle size and geometry.

\section{MATERIALS AND EXPERIMENTAL METHODS}

Three batches of fibers, as shown in Fig. 1, were manufactured from fiber optic filaments with poly(methyl methacrylate) cores. The filaments were soaked in dimethyl sulfoxide, mechanically wiped to remove the outer fluorocarbon coating, and then cut to the appropriate length to produce fibers with aspect ratios $A=L / d$ of $11 \pm 2$ and $23 \pm 2$, where $L$ and $d$ are the fiber length and diameter; two sets of fibers were used at $A=23$, where $L$ and $d$ of one set are double the other set. The fibers were suspended in a mixture of Triton X-100 (73\% by mass), distilled water (11\%), and zinc chloride $(16 \%)$. Fluorescent dye (rhodamine $6 \mathrm{G}$ ) was added to the mixture at a concentration of $9 \times 10^{-7} \mathrm{~g} / \mathrm{cm}^{3}$. This fluid was Newtonian, with a viscosity of $\mu=30 \mathrm{P}$, and the density $(\rho=1.24$ $\left.\mathrm{g} / \mathrm{cm}^{3}\right)$ and refractive index matched that of the fibers. These properties were characterized at the same temperature $\left(25^{\circ} \mathrm{C}\right)$ at which the experiments were performed.

The suspension was sheared within the custom-built cell shown in Fig. 2. Specifically, the fiber suspension was loaded in the region between the transparent belt [see Fig. 2(a)]. Pure suspending fluid was loaded into the space between the shear cell walls and the belt. Since the belt extends from the bottom of the shear cell to a point above the fluid, fibers were unable to escape the region between the belts, and the concentration of fibers was maintained constant throughout the experiment. The suspension was gently mixed and sheared, allowing trapped air to escape to the surface prior to each experiment.

The belt was held in place by applying tension with the aluminum cylinders at either end of the cell, and a shearing flow was generated by the relative motion of the transparent belt upon rotating the cylinders as indicated in Fig. 2(a). Grooves were etched into the top of the belt for the cylinders to grip and aid with rotating the belt. Gears on one of the cylinders were attached to a stepper motor to actuate the motion, while the other cylinder rotated freely. Spacers made of acrylic were inserted 

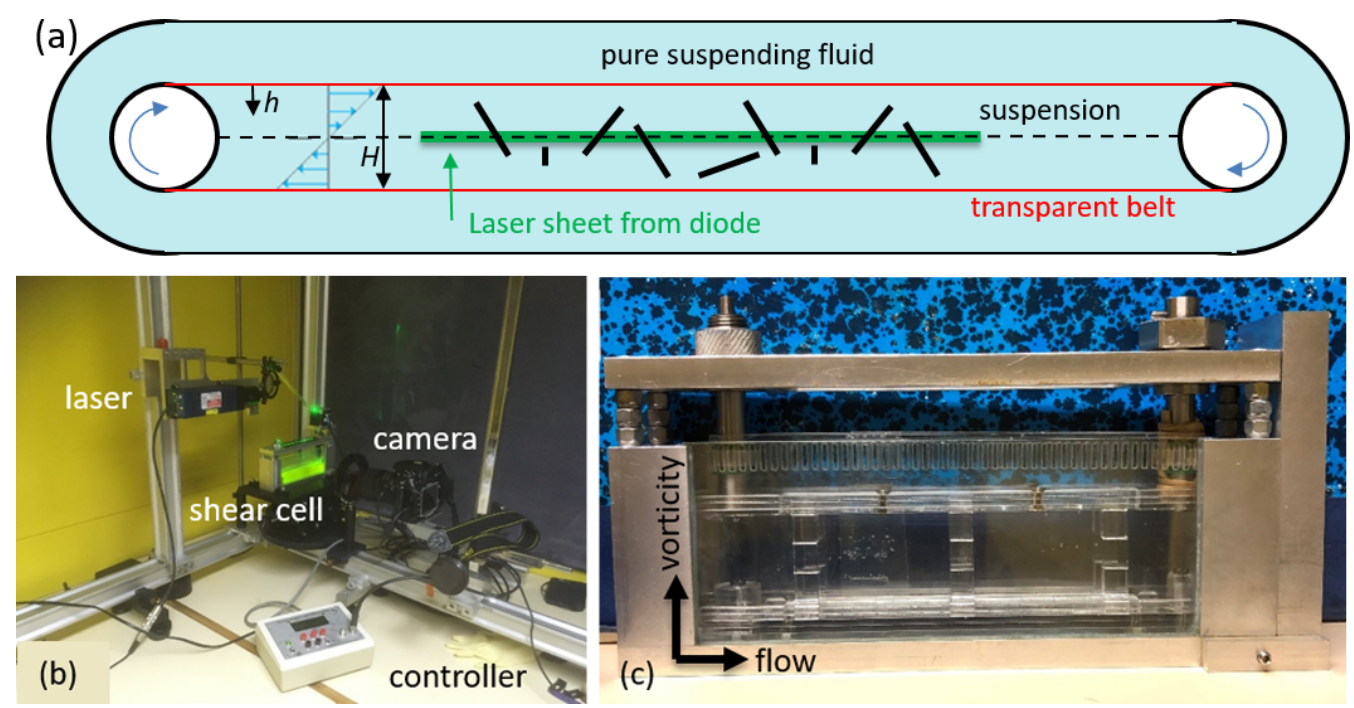

FIG. 2. (a) Schematic of the shear cell as viewed from above. The suspension is sheared by the transparent acrylic belt (red) which is driven by rotating the cylinders (white) at either end of the cell. A laser sheet (green) of thickness $250 \mu \mathrm{m}$, centered in the gap, fluoresces the fluid and enables imaging of the particle orientation distribution. (b) Photograph showing the controller and imaging equipment that surrounds the shear cell. A stepper motor attached to the cylinders drives the shearing flow, which is oscillated using a controller that also actuates the shutter and camera. (c) View of the shear cell from the same direction as the camera. The laser sheet fluoresces the suspension in the flow-vorticity plane.

in the shear cell to set the gap size at either $H=15.6$ or $7.8 \mathrm{~mm}$, which corresponds to $H=1.5 \mathrm{~L}$ for the fibers of shorter length $L=5.2 \mathrm{~mm}$. The length of the controlled gap (cylinder to cylinder) was $11 \mathrm{~cm}$, and the maximum height of fluid was $4.5 \mathrm{~cm}$.

A controller operated the stepper motor to generate an oscillatory shearing flow. The rate of shear was constant for the forward and backwards motions (i.e., a square wave), rather than a sinusoid, and the instantaneous strain increased from zero to a value of $2 \gamma_{0}$ before returning to zero for each cycle. The range of strain amplitudes $\gamma_{0}$, gap sizes $H$, volume fractions $\phi$, and fiber aspect ratios $A$ were chosen similar to the experimental work of Franceschini et al. [15] and computational work of Snook et al. [17]. The maximum shear rate of $1.67 \mathrm{~s}^{-1}$ used in the experiments gives a maximum particle Reynolds number of $\operatorname{Re}=\rho \dot{\gamma} L^{2} / \mu=0.07$, and Brownian motion is negligible owing to the large size of the fibers. The maximum stress exerted by the flow on the fibers was much smaller than the stress required to buckle a fiber [18]; consequently, the fibers can be considered rigid under the conditions of the experiment.

Figure 2(b) shows the spatial arrangement of the shear cell and surrounding imaging equipment. The laser sheet that entered the cell from the top [see Fig. 2(a)] had a width of $250 \mu \mathrm{m}$ and wavelength of $532 \mathrm{~nm}$. A shutter was mounted in front of the laser sheet to prevent photobleaching caused by overexposure of the fluid. The shutter and camera were actuated by the same controller that managed the oscillatory motion; at the end of each oscillatory cycle, a 5-s delay was implemented to minimize the free-surface deformation from the shear, and then one image was captured. Figure 2(c) shows the shear cell from the same direction as the camera, which allows for capturing images in the flow-vorticity plane through the transparent walls of the shear cell. Images were only captured in the flow-vorticity plane that is aligned with the center of the gap; the top metal piece in Fig. 2(c) restricts the imagining plane from various positions in the gap, such as capturing images near the wall.

Images collected during the experiments, such as the example in Fig. 3, were processed to calculate the orientation distribution. The fibers were marked, and the center of mass and orientations 

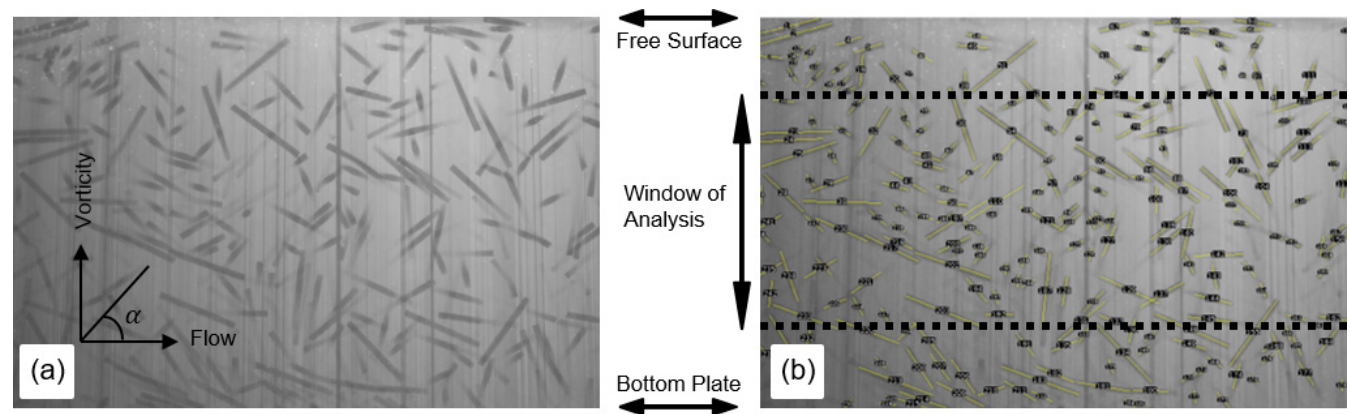

FIG. 3. (a) Example image showing fibers in the flow-vorticity plane, where $\alpha$ is the angle between the projection of the fiber in this plane with the flow direction. (b) Fibers within each image were identified and the orientation of each was determined. Only those particles at least one fiber length from the free surface and bottom plate were included in calculations of the orientation distribution.

were measured. To minimize any effects of the free surface and the bottom bounding wall of the apparatus, the window of analysis was limited to the region one particle length from each boundary. Quantitative information on the orientation distribution was extracted from the processed images. As depicted in Figs. 3(a) and 4(a), the angle $\alpha$ is the angle between the flow direction and the projection of the fiber in the flow-vorticity plane. The angle $\alpha$ was used in the computation of the order parameter $S_{\alpha}$, a measurement of the fiber orientation. Here, $S_{\alpha}=1-2\left\langle\cos ^{2}(\alpha)\right\rangle$, where the $\langle\cdot\rangle$ denotes an average over fibers in the window of analysis. Note that $S_{\alpha}=0$ for a suspension with a random orientation distribution, and $S_{\alpha}=-1$ or 1 indicates a suspension with perfect fiber alignment in the flow or vorticity direction, respectively.

\section{SIMULATIONS}

To assist in interpreting the experimental results and spanning a wider range of the dimensionless parameters, simulations were used. The model is similar to that used by Snook et al. [17] to simulate the oscillatory shear of concentrated suspensions of rigid fibers. The equations ignore the

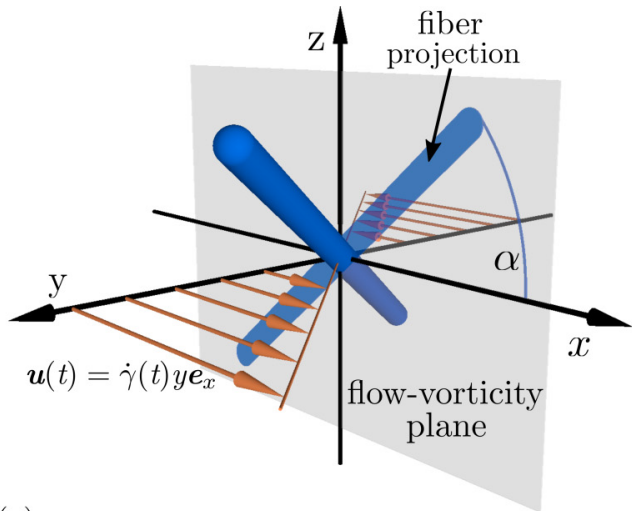

(a)

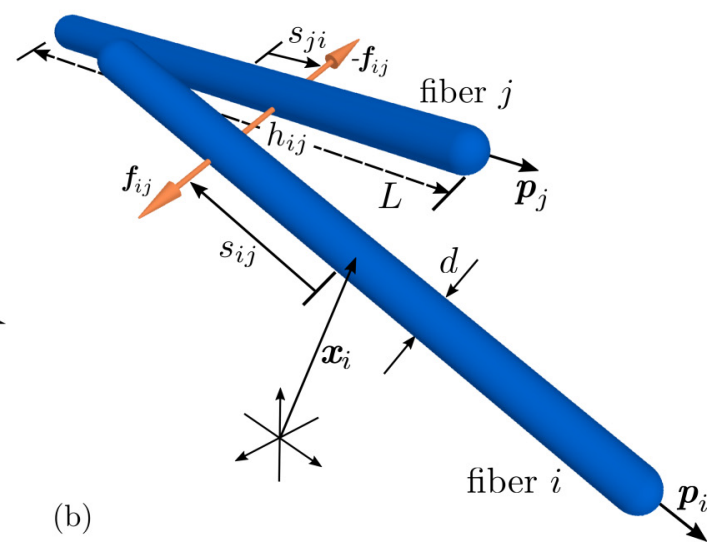

(b)

FIG. 4. (a) The $x, y$, and $z$ directions are the flow, gradient, and vorticity directions, respectively. The angle between the fiber projection in the flow-vorticity plane and the flow direction is $\alpha$. (b) Each fiber of aspect ratio $A$ is defined by its center-of-mass position $\boldsymbol{x}_{i}$ and orientation $\boldsymbol{p}_{i}$. A Hertzian repulsive force acts to separate pairs of fibers when their minimum separation distance $h_{i j}$ indicates an overlap [refer to Eq. (3)]. 
effects of inertia since the Reynolds number is small, and the hydrodynamic forces are modeled using slender-body theory for a rigid rod at leading order [19,20]. Furthermore, the model excludes hydrodynamic interactions between particles; this approximation has been used to predict accurately the orientation distribution of high-aspect ratio fibers at sufficiently large concentrations [21-23], as contact interactions between fibers predominately influence the dynamics.

Using the above assumptions and balancing the hydrodynamic drag and short-range repulsive forces $\left(\boldsymbol{F}_{i}\right)$ to maintain the excluded volume of the fibers gives

$$
\dot{\boldsymbol{x}}_{i}=\boldsymbol{u}\left(\boldsymbol{x}_{i}\right)+\xi^{-1}\left(\boldsymbol{I}+\boldsymbol{p}_{i} \boldsymbol{p}_{i}\right) \cdot \boldsymbol{F}_{i},
$$

where $\dot{\boldsymbol{x}}_{i}$ is the velocity of the center of mass $\boldsymbol{x}_{i}$, and $\boldsymbol{p}_{i}$ is the unit vector along the axis of fiber $i$ [see Fig. 4(b)]. An oscillatory shear flow, $\boldsymbol{u}\left(\boldsymbol{x}_{i}, t\right)=\dot{\gamma}(t) y \boldsymbol{e}_{x}$, is imposed, with flow in the $x$ direction and the gradient in the $y$ direction as defined in Fig. 4(a). The shear rate is a square wave of magnitude $\dot{\gamma}_{0}$ and the period is determined by the strain amplitude $\gamma_{0}$; simulations were also run using a sine-wave oscillation method, replicating the conditions of the previous work $[15,17]$. The coefficient for the mobility is $\xi^{-1}=\ln (2 A) / 4 \pi \mu L$.

Likewise, the rotational motion is governed by a balance between the torque due to hydrodynamic drag and torque caused by the repulsive forces between fibers $\left(\boldsymbol{T}_{i}\right)$,

$$
\dot{\boldsymbol{p}}_{i}=\boldsymbol{\Omega} \cdot \boldsymbol{p}_{i}+B\left(\boldsymbol{I}-\boldsymbol{p}_{i} \boldsymbol{p}_{i}\right) \cdot \boldsymbol{E} \cdot \boldsymbol{p}_{i}+\frac{12 \xi^{-1}}{L^{2}} \boldsymbol{T}_{i} \times \boldsymbol{p}_{i}
$$

In the above equation, rotation of a slender body due to a shearing flow has been replaced by the rotation of a rod with a finite thickness. The fiber rotates in proportion to the rotation of the fluid, $\boldsymbol{\Omega}=\frac{1}{2}\left[(\nabla \boldsymbol{u})-(\nabla \boldsymbol{u})^{T}\right]$, and a fraction $B=\left(A_{e}^{2}-1\right) /\left(A_{e}^{2}+1\right)$ of the extensional flow, $\boldsymbol{E}=$ $\frac{1}{2}\left[(\nabla \boldsymbol{u})+(\nabla \boldsymbol{u})^{T}\right]$. The parameter $B$ is a correction for the rotation of a spherocylinder [24-26] instead of an ellipsoid [27], using a fiber effective aspect ratio $A_{e}=0.8 \mathrm{~A}$, rather than the aspect ratio $A$; for these calculations, $A_{e}=8.8$ and 18.4 when the fibers are of aspect ratio $A=11$ and 23 , respectively.

To maintain the excluded volume of the particles, Snook et al. [17] applied a repulsive force between overlapping fibers that was constant. Here, a Hertzian force [28,29] was applied to prevent the overlap of two fibers in contact [see Fig. 4(b)],

$$
\boldsymbol{f}_{i j}^{(c)}= \begin{cases}0, & \text { if } h_{i j}>2 \epsilon, \\ k_{n}\left(\frac{h_{i j}}{2 \epsilon}\right)^{\frac{3}{2}} \boldsymbol{n}_{i j}, & \text { if } h_{i j} \leqslant 2 \epsilon,\end{cases}
$$

where $k_{n}=4 \pi \mu \dot{\gamma} L^{2} / \ln (2 A), h_{i j}$ is the minimum separation distance between two fibers $i$ and $j$, $\epsilon=0.1 d$ is the estimated roughness of a fiber in the experiments, and $\boldsymbol{n}_{i j}= \pm\left(\boldsymbol{p}_{i} \times \boldsymbol{p}_{j}\right) /\left|\boldsymbol{p}_{i} \times \boldsymbol{p}_{j}\right|$ is the normal direction of the collision. The sign of the normal is chosen so that the fibers repel, rather than attract, each other. Periodic boundaries were used in the flow and vorticity directions, and particles were maintained within the bounding walls using a similar forcing,

$$
\boldsymbol{f}_{i}^{(w)}= \begin{cases}0, & \text { if } h_{i}>\epsilon, \\ k_{n}\left(\frac{h_{i}}{\epsilon}\right)^{\frac{3}{2}} \boldsymbol{n}_{i}, & \text { if } h_{i} \leqslant \epsilon,\end{cases}
$$

where $h_{i}$ is the minimum separation between the fiber $i$ and the nearest wall, and the normal points from the wall into the fluid.

The summation of interactions for a fiber $i$ yields the total nonhydrodynamic force,

$$
\boldsymbol{F}_{i}=\boldsymbol{f}_{i}^{(w)}+\sum_{j=1}^{v_{T}} \boldsymbol{f}_{i j}^{(c)}
$$




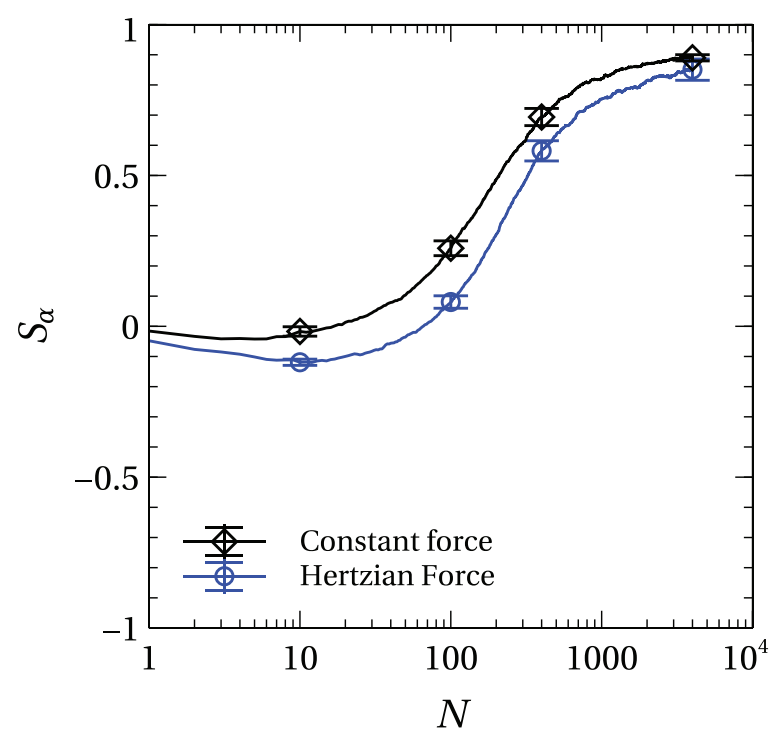

FIG. 5. The order parameter $S_{\alpha}$ as a function of the oscillation number $N$ of the simple contact force simulations used in previous calculations [17] compared to the change in the forcing to a Hertzian contact force. The conditions used were $H=1.5 L, A=11, \phi=0.20$, and $\gamma_{0}=2.5$; both curves were produced using the sine-wave oscillation method and the initial distributions are identical. Averaging is performed over all fibers within the gap and over 12 runs.

and torque,

$$
\boldsymbol{T}_{i}=s_{i} \boldsymbol{p}_{i} \times \boldsymbol{f}_{i}^{(w)}+\sum_{j=1}^{v_{T}} s_{i j} \boldsymbol{p}_{i} \times \boldsymbol{f}_{i j}^{(c)}
$$

where $s_{i j}$ is the point on fiber $i$ where it collides with fiber $j$, as illustrated in Fig. 4(b), and $s_{i}$ is the point on fiber $i$ where it collides with the wall. The sums over the total number of fibers $v_{T}$ exclude when $i=j$.

Equations (1) and (2) were integrated in time using the Euler method, starting from an initial set of particle positions and orientations. The initial distribution for each simulation was created by randomly placing particles in the simulation box, while rejecting any placements that generated an overlap. Simulations were performed using up to $v_{T}=5052$ particles (given a box size of $5 L \times 3 L \times 5 L$ ), which varies depending on box size, aspect ratio $A$, and concentration $\phi$. The time step was set to a value of $\Delta t=0.001 L /\left(H \dot{\gamma}_{0}\right)$, which ensured that displacements were smaller than the range over which the repulsive force operates. The computational expense, caused by the combination of small time steps and total time over which simulations were run, was mitigated in part by making use of link lists [30] to facilitate the search for collisions between particles.

Figure 5 compares $S_{\alpha}$ calculated using the constant repulsive force model used by Snook et al. [17] and the Hertzian force model [see Eqs. (3) and (4)]. Twelve runs were performed for $H=1.5 \mathrm{~L}$, $A=11, \phi=0.20$, and $\gamma_{0}=2.5$ using a sine-wave oscillation. The values of $S_{\alpha}$ were determined by averaging over all fibers in the simulations within the gap, and the error bars shown in the figure represent the standard deviation of the mean across the 12 runs. Given identical initial conditions, $S_{\alpha}$ is slightly higher for the constant repulsive force model than the Hertzian force model following each oscillation $N$, though the steady-state result is the same within the calculated error. The predictions for $S_{\alpha}$ are similar since the range over which the forces operate $(h<2 \epsilon)$ are identical. 

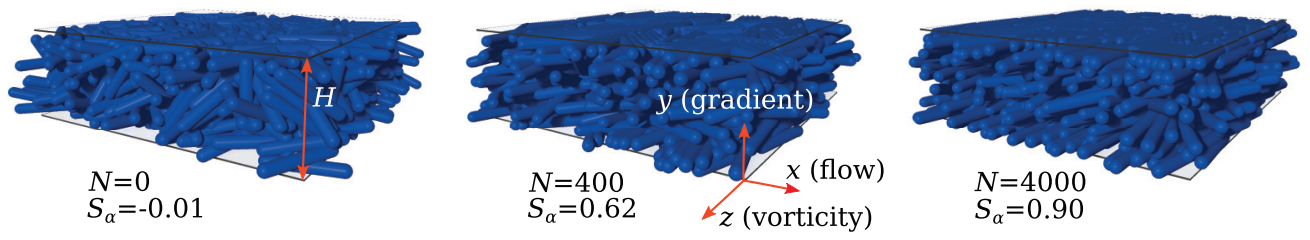

FIG. 6. Visualization of an initial condition for the fibers and their predicted distribution after $N=400$ and 4000 oscillations. Fibers generally rotated into alignment with the vorticity direction for this set of conditions, which consisted of $H=1.5 L, A=11, \phi=0.20$, and $\gamma_{0}=2.5$ with a square-wave oscillation. Vorticity alignment is reflected by the increased values of $S_{\alpha}$, which were calculated using all fibers in the gap.

\section{RESULTS AND DISCUSSION}

Section IV A describes simulation results that indicate spatially dependent alignment of the fibers, and Sec. IV B compares experimental and numerical results from this work with previous ones. Later sections present results on the larger range of conditions. Including the gap $H$, a total of four length scales $\left(L, d\right.$, and strain displacement $\left.\gamma_{0} H\right)$ were varied in the experiments. Additionally, the fiber concentration was varied and is reported as either a volume fraction $\phi$ or a dimensionless number density $n L^{2} d=4 A \phi / \pi$; the number of fibers normalized by the total volume is the number density $n$. Note that the alignment is assumed to depend only on the gap $H$, and not the length and height of the test cell. Also, the alignment depends on the number of oscillations $N$, but not on time. Time does not enter into the problem directly since the flow was in the creeping regime and there were no other timescales due to the absence of Brownian motion and gravitational effects (i.e., the particle and fluid densities matched). Consequently, the alignment $\left(S_{\alpha}\right)$ at any value of $N$ is expected to be a function of four dimensionless parameters $\left(A, \gamma_{0}, H / L\right.$, and either $\phi$ or $\left.n L^{2} d\right)$ constructed from the five variables and one independent unit (length).

\section{A. Spatial dependence of the order parameter}

Figure 6 illustrates a set of fibers, starting from the initial distribution $(N=0)$, and after simulating $N=400$ and 4000 oscillations using the methods presented in Sec. III. The order parameters $S_{\alpha}$ reported in the figure were evaluated by averaging over all fibers in the gap; it was found to increase from the initial value of $S_{\alpha}=-0.01$ to 0.79 , indicating a preferential fiber alignment in the vorticity direction after 4000 cycles. Correspondingly, the images indicate that the fibers tend to align in the vorticity direction throughout the gap, though alignment is a function of position as seen more explicitly in Fig. 7(a). This figure shows slices of width $d / 2 L$ at the center of the gap and adjacent to a bounding wall for the same set of data as in Fig. 6. Initially, fibers near the wall lie mostly in the flow-vorticity plane with no preferential alignment, while many of the fibers that intersect the flow-vorticity plane in the center of the gap extend substantially in the gradient direction. Additionally, the number of fibers intersecting the center slice exceeds the number in the wall-adjacent slice. After oscillating 400 and 4000 times, fibers near the wall and in the center of the gap aligned more fully in the flow-vorticity plane and preferentially oriented in the vorticity direction. Notably near the wall, fibers organized more strongly than in the center, and the relative number of fibers near the wall surpassed the number in the center of the gap after oscillating approximately 100 times.

The order parameter $S_{\alpha}$ was evaluated as a function of oscillation number and was averaged over multiple simulations. Figure 7(b) shows the results for the bulk value and for fibers in the center and adjacent to the wall. Note that bulk values of $S_{\alpha}$ were produced by averaging over all fibers in the gap, while the center slice and wall slice values of $S_{\alpha}$ were calculated using only those fibers intersecting a slice of thickness $d / 2 L$ at the center and adjacent to the wall, respectively. As $N$ increased to 4000 cycles, the bulk $S_{\alpha}$ first dropped to a minimum value of $-0.32 \pm 0.03$ at $N \approx$ 


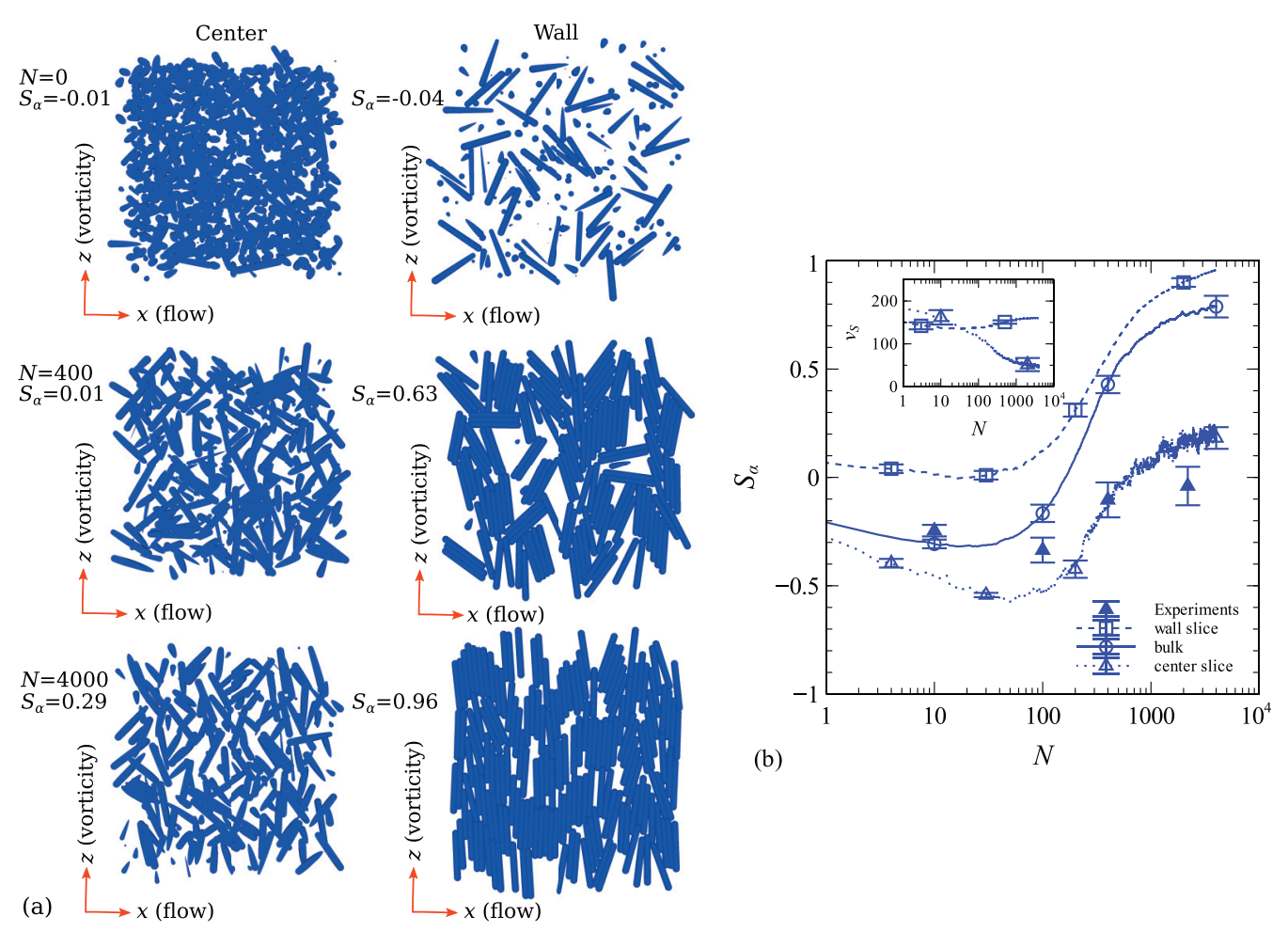

FIG. 7. Results from simulations with $H=1.5 L, A=11, \phi=0.20, \gamma_{0}=2.5$, and a square-wave oscillation. (a) Illustrations of slices of the suspension at the center of the gap (left column) and near the wall (right column) evolving from an initial state $(N=0)$ through the steady-state structure at $N=4000$. Reported values of $S_{\alpha}$ were calculated only over those fibers intersecting each slice. (b) The order parameter $S_{\alpha}$ as a function of the number of oscillations $N$ for the square-wave model presented in Sec. II using every fiber in the gap (circles), fibers intersecting the center (open triangles), and fibers within a diameter of the wall (square symbols). Order parameters measured from experiments are also shown (filled triangles). The inset shows the calculated average number of fibers $v_{S}$ present in the wall and center slices as a function of $N$ from the square-wave model. Error bars represent the standard deviation of the mean values calculated from multiple realizations of either the simulations or experiments.

25 before increasing and approaching a steady-state value of $0.79 \pm 0.03$. This set of conditions ( $\left.H=1.5 L, A=11, \phi=0.20, \gamma_{0}=2.5\right)$ gave the largest bulk value of the order parameter across all conditions that were studied. The order parameter for the wall and center slices remain larger and smaller, respectively, than the bulk value.

The steady-state distribution was highly vorticity aligned for the wall slice $\left(S_{\alpha}=0.96\right)$, and the fibers in the center of the gap began to align more in the flow direction $(N \approx 100)$ before weakly aligning in the vorticity direction $\left(S_{\alpha}=0.19\right)$ only after 500 oscillations. The numerical data in the center slice agree most closely with the experimental measurements. The inset of Fig. 7(b) shows the number of fibers $\left(v_{S}\right)$ intersecting slices at the center and adjacent to the wall as a function of oscillation number $N$ for the simulations. The number of fibers decreased in the center plane as fibers rotated out of the gradient direction and into the flow-vorticity plane; fibers near the wall self-organized as the concentration of fibers increased.

\section{B. Comparisons with previous work}

Figure 8 compares measurements reported by Franceschini et al. [15] [see Fig. 8(a)] to measurements from the experiments described in Sec. II [see Fig. 8(b)] over a range of strain amplitudes 

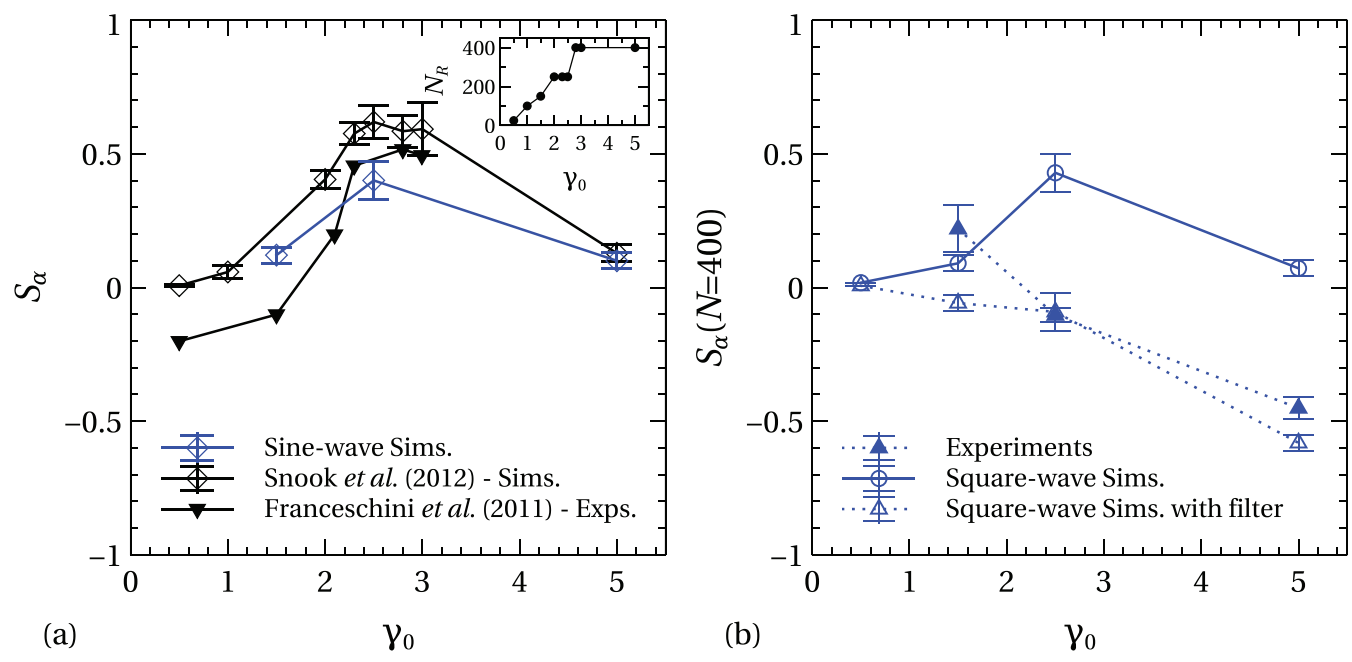

FIG. 8. Experimental and simulation results for $H=1.5 L, A=11$, and $\phi=0.20$. (a) The bulk order parameter $S_{\alpha}$ measured in previous experiments [15] is compared to simulation results, including those of Snook et al. [17]. The inset indicates the oscillation number $N_{R}$ at which $S_{\alpha}$ was determined for each $\gamma_{0}$. (b) The order parameter $S_{\alpha}$ measured from experiments are compared to simulations using square-wave oscillations. Simulations show $S_{\alpha}$ as calculated using all fibers in the gap, though the open triangles and dashed line used only those fibers intersecting the center plane of the gap when calculating $S_{\alpha}$.

and identical conditions of $H=1.5 L, A=11$, and $\phi=0.20$. Previous measurements indicate fibers strongly align with the the vorticity direction at $\gamma_{0} \approx 2.5$, where the order parameter $S_{\alpha}$ is maximized. Experimental results in Fig. 8(b) appear to show a very different dependence of $S_{\alpha}$ on $\gamma_{0}$; as $\gamma_{0}$ increases above $1.5, S_{\alpha}$ not only decreases, but is also negative, indicating an increased alignment of particles in the flow direction. The discrepancy in the results is due primarily to the spatial positions over which the orientation distributions were sampled and, to a lesser extent, the pattern of the shearing flow.

The laser sheet fluoresces the flow-vorticity plane in the center of the gap in the current experimental work, and the alignment of the fibers is position dependent as demonstrated in Figs. 6 and 7. Hence, the experimental data for $S_{\alpha}$ shown in Fig. 8(b) compare well with simulation results where only those fibers that intersect the center plane have been used in the evaluation. Similar to the experimental data, the simulations indicate smaller values of $S_{\alpha}$ as the strain amplitude increases, with quantitative agreement for $\gamma_{0}>1.5$. Analysis of simulation results that include all fibers when determining $S_{\alpha}$, which are also shown in Fig. 8(b), indicate much larger values than seen in the experiments. Likewise, Fig. 7(b) shows that the experimentally measured values of $S_{\alpha}$ as a function of oscillation number are much better predicted by the simulated values of $S_{\alpha}$ from fibers in the middle of the gap than across the entirety of the gap, as expected given the measurement position.

In the experiments by Franceschini et al. [15], fibers were sheared in a Couette cell and the orientation distribution was measured for fibers intersecting a laser sheet. Few details were given regarding the exact position and size of the laser sheet, but the curvature of the Couette cell prevented aligning the sheet uniformly in the flow-vorticity plane, and fibers not in the center of the gap were sampled as well. Figure 8(a) compares the order parameter measured by Franceschini et al. [15] with the present simulations and those of Snook et al. [17] using different contact forces but calculating $S_{\alpha}$ using all fibers in the gap; the inset indicates the oscillation $N_{R}$ at which $S_{\alpha}$ was determined for each $\gamma_{0}$ across all of the results shown. At the lower strain amplitudes $\left(\gamma_{0}=0.5-1.5\right)$, discrepancies between the simulations and the experiments are most likely due to different initial configurations, as the simulations used initially random orientations $\left(S_{\alpha} \approx 0\right)$ and the experiments were prealigned 

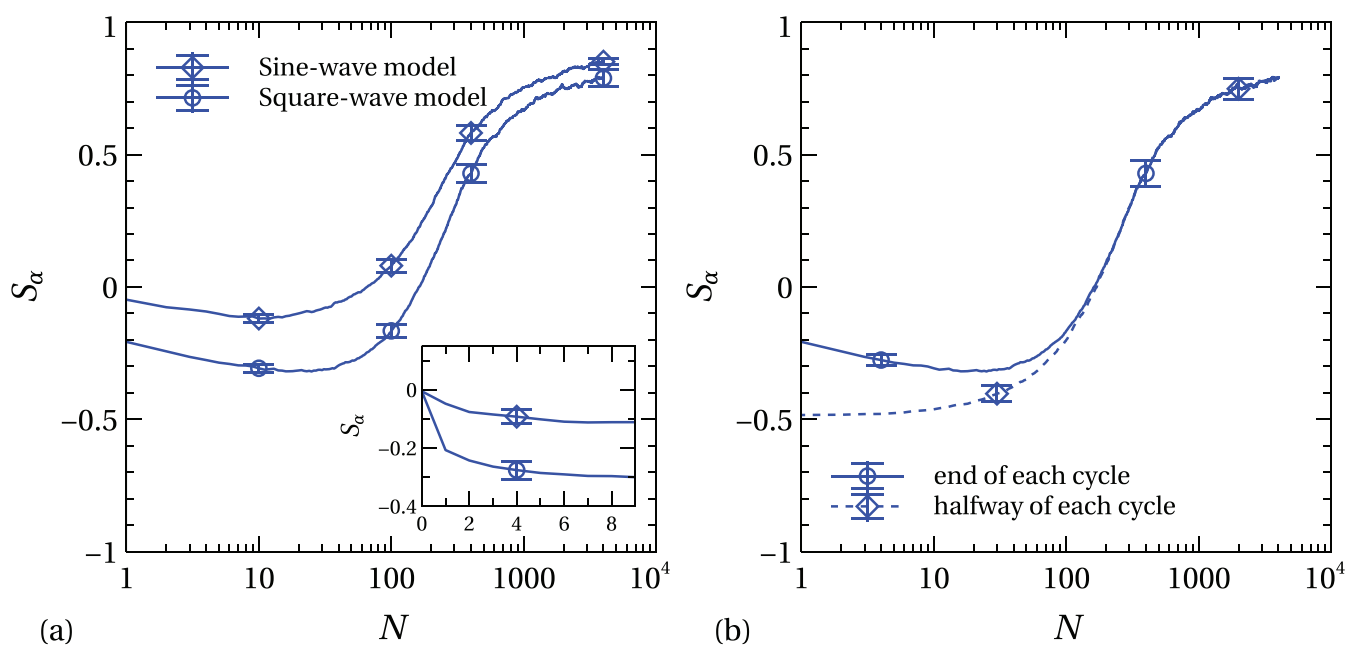

FIG. 9. Bulk values of $S_{\alpha}$ at $H=1.5 L, A=11, \phi=0.20$, and $\gamma_{0}=2.5$ from simulations. (a) The order parameter $S_{\alpha}$ as a function of the number of oscillations $N$ is different for the sine-wave and square-wave patterns of shear and measurement, and the inset shows the first nine cycles. (b) The order parameter as a function of $N$ when using the square-wave and recording the orientation distribution at end and half point of each cycle.

slightly in the flow direction $\left(S_{\alpha} \approx-0.20\right)$. The good agreement suggests that the measurements of Franceschini et al. [15] sampled fibers across the entirety of the gap.

Though the spatial sampling of fibers is the primary reason that the experimental results in Figs. 8(a) and 8(b) should not be compared directly, the pattern of oscillation and data collection also causes some differences that affect any comparisons with the work of Franceschini et al. $[15,16]$. Their experiments and the corresponding simulations of Snook et al. [17] utilized a sinusoidal strain displacement, where the instantaneous strain was increased from zero to a value of $\gamma_{0}$, was decreased to $-\gamma_{0}$, and then returned to zero, at which point the orientation distribution was sampled. The order parameter $S_{\alpha}$ from the previous works are compared in Fig. 8(a) to our simulations using the same sine-wave oscillation, though simulations results in Fig. 8(b) used a square-wave oscillation and sampling in order to mimic the experiments. For the square wave, the instantaneous strain is increased to $2 \gamma_{0}$ and then returned to 0 , at which point the measurement of orientation was made.

Using identical initial conditions, simulations shown in Fig. 9(a) demonstrate that the bulk value of $S_{\alpha}$ is lower at each value of $N$ when using a square-wave, as opposed to sinusoidal, pattern of shear and measurement. At $N=4000$ oscillations, the order parameter for the sine-wave simulations is $0.83 \pm 0.01$ and for square-wave simulations is $0.79 \pm 0.03$. The order parameter $S_{\alpha}$ also depends on when it is calculated throughout an individual cycle; evolution of $S_{\alpha}$ as a function of $N$ for various positions in the square-wave cycle is shown in Fig. 9(b) for square-wave oscillations. Until around $N=200$, the order parameter is consistently larger at the end of each cycle (dotted line) compared to the calculations recorded halfway through the cycle (solid line), the point at which the flow is reversed in the cycle. Similar differences in the $S_{\alpha}$ due to the oscillation methods were observed for all conditions in the current work.

Having established, with the aid of simulations, that the experiments performed here are consistent with those of Franceschini et al. [15], the remaining section presents a wider range of conditions. The remaining simulation and experimental results use only the square-wave pattern of oscillation and sampling, except where noted. 


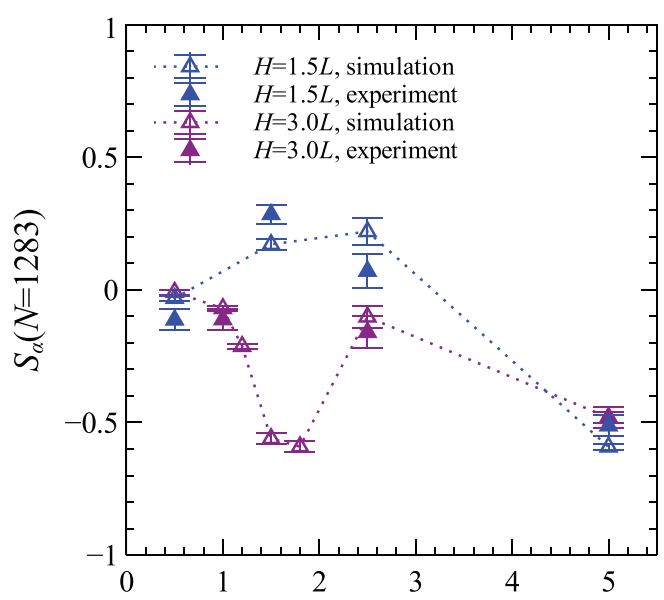

(a)

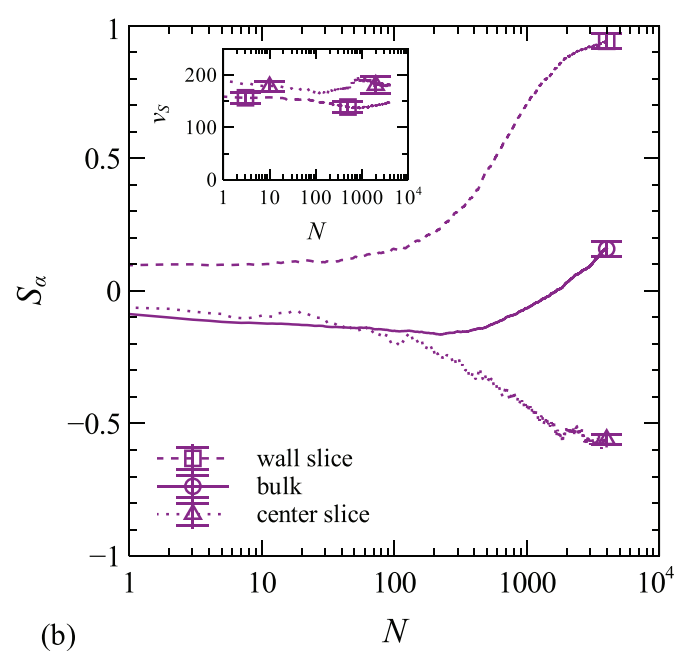

(b)

FIG. 10. Calculations and measurements performed with $A=11$ and $\phi=0.20$. (a) The order parameter $S_{\alpha}$ was evaluated for fibers intersecting the center of the gap for simulations and experiments at different $H$ and $\gamma_{0}$; here, $S_{\alpha}$ was measured at $N=1283$, the largest common number of oscillations across every experiment and simulation. (b) The bulk, center, and wall-adjacent values of $S_{\alpha}$ as a function of $N$ for $H / L=3.0$ and $\gamma_{0}=1.5$. The inset shows the calculated average number of fibers $v_{S}$ that intersect the center and wall plane as a function of $N$.

\section{Orientation dependence on gap size, aspect ratio, and concentration}

The effect of confinement on the order parameter is depicted in Fig. 10, where $A=11$ and $\phi=0.20$. The order parameter of the final cycle recorded in the experiments compares well with simulation results, as shown in Fig. 10(a); only fibers intersecting the center of the gap were included in the calculation of $S_{\alpha}$ and the measurements and simulations are reported at $N=1283$, the largest common oscillation number among the experiments shown in the figure. For the smaller $H / L$ of 1.5 , fibers at the center of the gap orient only slightly in the direction of vorticity at intermediate strain amplitudes at $N=1283$. Fibers at the center of the gap align preferentially in the gradient direction for $H / L=3.0$, though a surprising dip in $S_{\alpha}$ is predicted by the simulations at $\gamma_{0} \approx 1.5$. Figure 10(b) explores the dynamics for this condition and shows that $S_{\alpha}$ for fibers in the center diverges from the bulk value after approximately 100 oscillations, with the fibers in the middle turning towards the flow direction while fibers adjacent to the wall strongly align with the vorticity direction. The inset of Fig. 10(b) reports that the calculated average number of fibers in each of the center and wall slices remains relatively constant throughout the development of the microstructure, much unlike the conditions presented in the inset of Fig. 7(b).

Bulk values of $S_{\alpha}$ were evaluated from simulations at $N=4000$ for gap sizes between $H=1.5$ and 3.0 for a range of $\gamma_{0}$ values; for all but the smallest strain amplitude of 0.5 , the orientation distribution was steady by $N=4000$. Figure 11(a) shows that each confinement produces a maximum peak value in the bulk $S_{\alpha}$ at $\gamma_{0}=2.5$, with the highest being $S_{\alpha}=0.79 \pm 0.03$ at $H=1.5 \mathrm{~L}$. As the confinement relaxes to $H=3.0 L, S_{\alpha}$ drops to $0.50 \pm 0.04$. At $\gamma_{0}=2.5$, alignment in the vorticity direction is remarkably similar as a function of distance from the wall $(h / L)$ across all of the studied gaps [see Fig. 11(b)]. Fibers adjacent to the wall are strongly aligned in the vorticity direction, with $S_{\alpha}>0.9$. The orientation distribution returns toward a random alignment $\left(S_{\alpha}=0\right)$ as the distance $h / L$ from the wall increases. Note that the inset of Fig. 11(a) shows that the peak values of $S_{\alpha}$ scale as $(H / L)^{-0.6}$, though calculating this result from $S_{\alpha}(h / L)$ requires consideration of the position-dependent concentration. This is shown in the inset of Fig. 11(b) and indicates that 


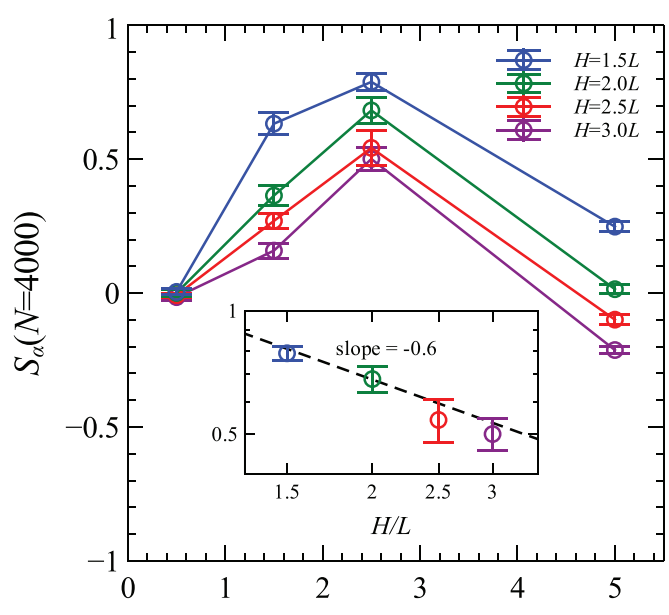

(a)

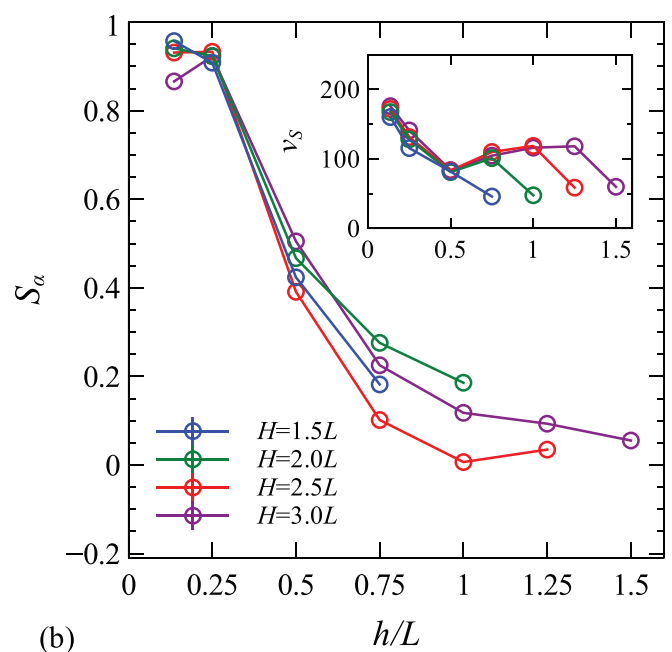

FIG. 11. Simulation results for $A=11$ and $\phi=0.20$. (a) The bulk order parameter $S_{\alpha}$ was evaluated for multiple gap sizes $H / L$ at $N=4000$ across multiple $\gamma_{0}$. The inset shows the maximum $S_{\alpha}$ for each gap size $H$ (occurs at $\gamma_{0}=2.5$ ) as a function of $H / L$. (b) Spatial variation of $S_{\alpha}$ for various $H$ when $\gamma_{0}=2.5$, where $h / L$ is the distance from the wall [see Fig. 2(a)]. The inset shows the calculated average number of fibers $v_{S}$ as a function of $h / L$.

the fibers are more concentrated near the wall where the fibers are highly organized and aligned with the vorticity direction.

Two sets of fibers with an aspect ratio of $A=23$ were used in experiments, as pictured in Fig. 1, where both the length and diameter of one set is roughly double the second set. Experimental measurements for both sets of $A=23$ fibers are compared in Fig. 12 to simulation results at the same confinement of $H=1.5 \mathrm{~L}$ and oscillation number $N=1395$, the largest common oscillation number between experiments; to compare the measurements, $S_{\alpha}$ from the simulations was calculated only for fibers intersecting the midplane between the bounding walls. The experiments and simulations show slightly reduced values of $S_{\alpha}$ at $\gamma_{0}=2.5 \mathrm{vs} 1.5$, with the results indicating that the alignment is close to random in the center and across the gap of the channel as well. The experimental results should be the same, regardless of the physical size of the fibers since all other parameters $(H / L$, $\gamma_{0}$, and $\phi$ ) were held constant. However, the values of $S_{\alpha}$ are larger for the bigger fibers, and the reported measurement errors do not account for the differences. One notable possibility is that the height of the fluid in the cell may have impacted the results.

To further assess the functional dependence of alignment on aspect ratio and concentration, simulations were performed for two different aspect ratios and concentrations over a range of strain amplitudes. The results for the predicted bulk values of $S_{\alpha}$ are given in Fig. 13(a) along with some experimental results of Franceschini et al. [16] for a concentration of $\phi=0.10$. Similar to the simulation results at the same conditions, a maximum $S_{\alpha}$ is observed around $\gamma_{0}=4-5$ in the experiments, though it should be noted that the comparison is at a different oscillation number and the experiments were performed using a sine-wave oscillation. The simulation data in Fig. 13(a) demonstrate that there is no simple agreement when comparing order parameters on the basis of either volume fraction $\phi$ or dimensionless number density $n L^{2} d$. At the same particle aspect ratio $A=11$, a decrease in the concentration by a factor of 2 (from $\phi=0.20$ to 0.10 or from $n L^{2} d=2.88$ to 1.44) yields distinctive fiber orientations, even though both are considered concentrated suspensions; the higher concentration system $\left(\phi=0.20\right.$ or $\left.n L^{2} d=2.88\right)$ indicates a strong alignment in the vorticity direction $\left(S_{\alpha}=0.79\right.$ at $\left.N=4000\right)$, whereas the lower concentration system $(\phi=0.10$ or $\left.n L^{2} d=1.44\right)$ indicates increasing alignment in the flow direction $\left(S_{\alpha}=-0.35\right.$ at $\left.N=4000\right)$. 


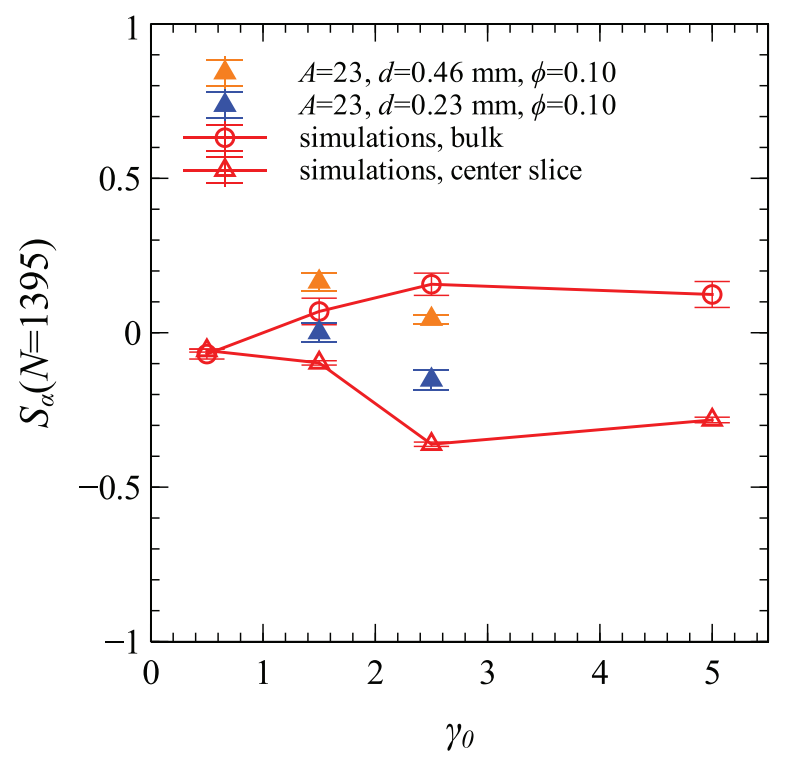

FIG. 12. Measurements and simulations with $A=23, H / L=1.5$, and $\phi=0.10$. The order parameter $S_{\alpha}$ from experiments (solid symbols) is compared to simulation results where $S_{\alpha}$ was evaluated for fibers intersecting the center of the gap (open symbols). The results are reported at the common terminal oscillation $N=1395$ of the experiments. The bulk order parameter $S_{\alpha}$ evaluated from simulation results is also shown.

Simulation results for $A=23$ indicate little vorticity alignment relative to $A=11$ when comparing both at identical $\phi$ and $n L^{2} d$.

The orientation distribution is controlled by contacts between particles and the bounding walls, as indicated by the simulations by Snook et al. [17] and here. As the strain amplitude is increased at a

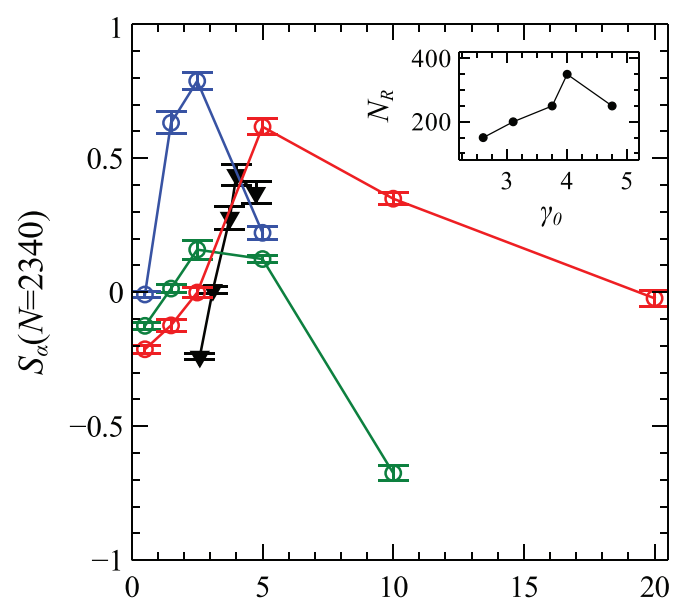

(a)

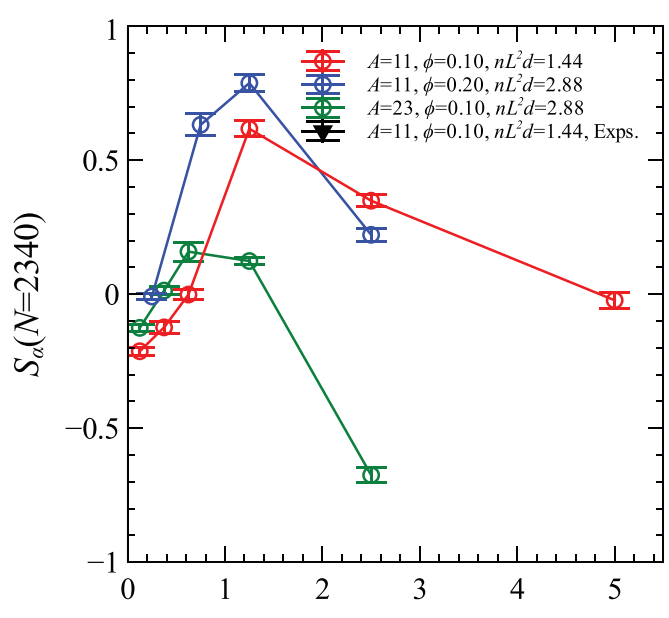

(b)

$\gamma_{0} \phi / \Phi_{c}$

FIG. 13. (a) Bulk values of $S_{\alpha}$ from simulations for $N=2340, H=1.5 L$, and $A=11$ and 23 for a range of concentrations. Some experimental results [16] are also shown for comparison; the inset indicates the oscillation number at which the experimental data were reported. (b) Simulations results for the bulk values of $S_{\alpha}$ are replotted by scaling the strain amplitude by $\phi / \Phi_{c}$, where $\Phi_{c}=0.4$ as found by Franceschini et al. [16] from rheological measurements. 

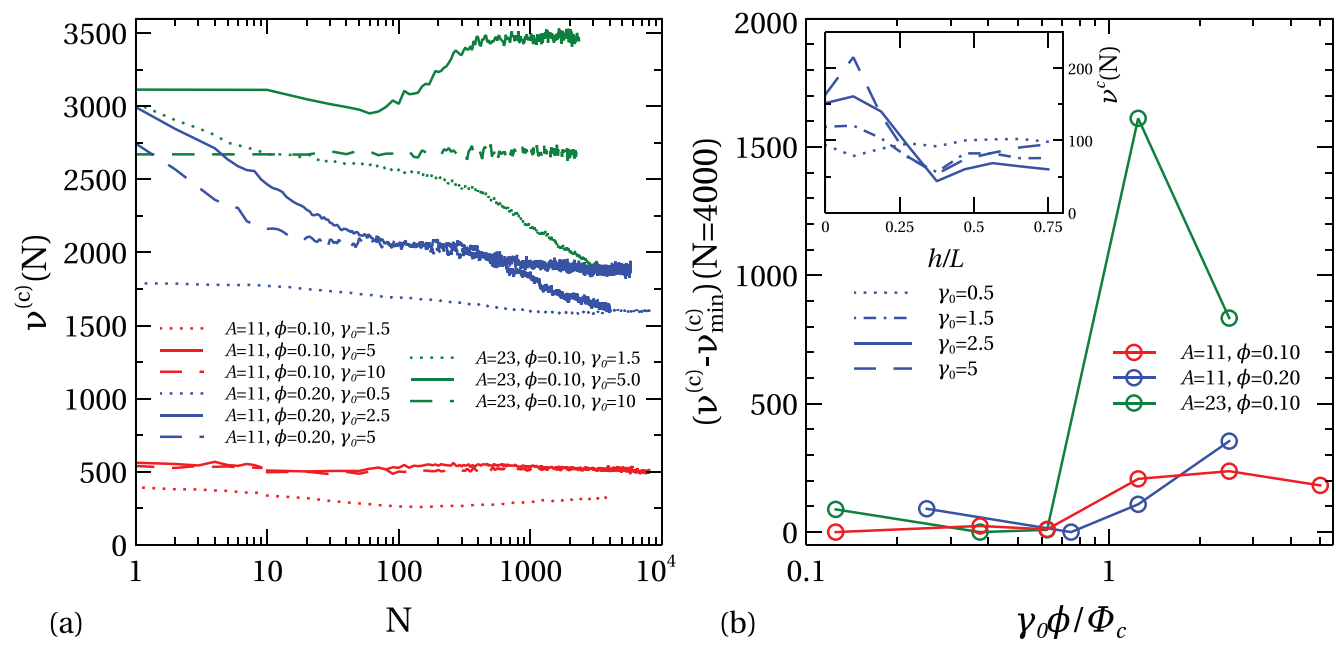

FIG. 14. Average number of contacts $\left(v^{(c)}\right)$ extracted from simulations with $H=1.5 L$ and $A=11$ and 23 . (a) The total number of fiber contacts with other fibers and bounding walls as a function of the oscillation number $N$. Results below, near, and above the critical rescaled strain amplitude of $\gamma_{0} \phi / \Phi_{c}=1$ are shown for volume fractions $\phi=0.10$ and 0.20 . (b) The total number of contacts are compared at $N=4000$, after having subtracted the minimum value of $v^{(c)}$ at $N=4000$ for each volume fraction. The number of contacts markedly increases at $\gamma_{0} \phi / \Phi_{c} \approx 1$. The inset shows the spatial distribution of contacts for $A=11$ and $\phi=0.2$, where $h / L$ is the distance from the bounding wall.

fixed concentration, initially random rods experience more collisions during a cycle; below a critical strain amplitude, particles organize into a state that significantly lowers the number of contacts, or "activity" as defined by Franceschini et al. $[15,16]$. Likewise, at a fixed strain amplitude, a critical concentration exists at which collisional activity transitions. Data from oscillatory rheology were used to conclude an inverse relationship between the critical strain and concentration at which the activity transitions. Assuming that the transition to and from vorticity alignment scales in a similar manner, the data for $S_{\alpha}$ in Fig. 13(b) were replotted by a rescaled strain amplitude of $\gamma_{0} \phi / \Phi_{c}$. Here, $\Phi_{c}=\gamma_{c} \phi$ is the critical volume fraction as defined by Franceschini et al. [16], which represents the effective volume fraction swept out by the fibers rotating in the shear flow of amplitude $\gamma_{c}$. We use the value of $\Phi_{c} \approx 0.4$ that they found for fibers at high concentration. As shown in the inset of Fig. 13(b), the data for $A=11$ collapse for this choice of scaling, with the maximum close to a value of one. For $A=23$, the success of the scaling is not so clear, as the maximum value of $S_{\alpha}$ is not as prominent as for data with $A=11$. Also, $\Phi_{c}$ is likely a function of the aspect ratio.

\section{Contact interactions}

Since contact forces drive the alignment of fibers, significant changes in the contact interactions must occur as the order parameter $S_{\alpha}$ increases to its maximum value at $\gamma_{0} \phi \approx \Phi_{c}$. Extracting information on the contacts from the experiments is not directly possible, but simulation results on the contacts are given in Fig. 14(a). The average number of contact interactions $\left(v^{(c)}\right.$, evaluated at the completion of each oscillation) is shown as a function of the oscillation number. The contacts include those between fibers and between fibers and the bounding walls, and the number of contacts is generally larger at higher concentrations and at higher aspect ratios.

The number of contacts remains nearly constant as a function of $N$ for many of the conditions shown in Fig. 14(a), though other cases show a general decline or increase in the number of contacts. For strain amplitudes and concentrations below $\Phi_{c}$, Franceschini et al. [16] asserted that fibers 
organize into a reversible state where every particle returns to its position and orientation after each oscillation. In a reversible state, there would be no contacts. However, simulations indicate that the number of contacts do not decay to zero for any of the conditions studied here, and the positions and orientations of individual fibers were not reversible even for $\gamma_{0} \phi<\Phi_{c}$.

Despite the lack of a transition between a reversible and irreversible state at $\Phi_{c}$, there is a qualitative change in the orientation distribution [see Fig. 13(b)]. This change in $S_{\alpha}$ is accompanied by a change in the number of contacts, as shown in Fig. 14(b) which compares the number of contacts at $N=4000$. The number of contacts are relatively low and constant up until the product $\gamma_{0} \phi \approx \Phi_{c}$, indicating that the jump in the number of contacts coincides with maximum vorticity alignment and transition between viscous and elastic responses for the oscillatory rheology [16].

The inset of Fig. 14(b) shows the number of contacts versus distance from the bounding wall $(h / L)$ for $A=11, \phi=0.2$, and a range of strain amplitudes. Data at $h / L=0$ represent the number of particle contacts with the walls, whereas data for $h / L>0$ indicate the local number of contacts between particles. The wall contacts are nonzero even when the particles are highly aligned in the vorticity direction. For all but the lowest strain amplitude of $\gamma_{0}=0.5$, the number of contacts between particles is smaller in the center than near the bounding walls where vorticity alignment is stronger. Intuitively, higher alignment of fibers in the vorticity direction is expected to generate fewer contacts, at least for fibers suspended at a fixed concentration. This is because fibers aligned in the vorticity direction can simply rotate around their major axis without tumbling in the shear flow. However, the stronger vorticity alignment near the wall enables more particles to crowd into this space (see Fig. 7, for example). This higher concentration of particles explains the larger number of collisions between particles in the vicinity of the walls.

\section{CONCLUSIONS}

Results demonstrating the vorticity alignment of rods having an aspect ratio of 11 by Franceschini et al. [15,16] and Snook et al. [17] have been expanded to a wider set of conditions than previously studied. Here, both the aspect ratio of the fibers and their confinement were varied, and the effects on the orientation distribution have provided some insights into the vorticity alignment of the fibers.

The gap size in our experiments and simulations ranged from $H / L=1.5$ to 3.0, whereas Snook et al. [17] simulated two levels of confinement $(H / L=1.5$ and fully periodic boundary conditions). In addition to affirming that bulk measurements of vorticity alignment increase as confinement is increased, the orientation was found to be a strong function of distance from the wall. Indeed, demonstrating that the experimental measurements reported here match those of Franceschini et al. $[15,16]$ required consideration of the spatial dependence of the orientation distribution, since previous measurements sampled across the channel width and the measurements performed here sampled only the plane centered between the bounding walls. In particular for those conditions that maximized the bulk order parameter $\left(\phi=0.2\right.$ and $\left.\gamma_{0}=2.5\right)$, the wall-adjacent fibers aligned nearly perfectly with the vorticity direction regardless of the confinement. Also fibers concentrated near the wall as they self-organized, and the order parameter decayed toward $S_{\alpha}=0$ with distance from the wall, indicating a randomized orientation in the flow-vorticity plane. The higher concentration near the wall results in a larger number of contacts between particles than far from the wall, despite the stronger alignment of the particles in the vorticity direction.

The present work confirms that collisions between fibers suspended at high concentration drive their alignment in the vorticity direction, as suggested originally by Snook et al. [17]; the simulations correctly predict the measured orientations while only considering contributions to the motion of the fibers from collisions and self-mobilities. However, despite the simple contact origin of the phenomenon, vorticity alignment is a complex function of the confinement, fiber concentration and aspect ratio, and strain amplitude. The higher aspect ratio rods $(A=23)$ were found to only weakly align in the vorticity direction, and maximum alignment was observed at $H=1.5 L, A=11, \phi=$ 0.20 , and $\gamma_{0}=2.5$. Maxima in the bulk $S_{\alpha}$ over a range of conditions were found to occur at roughly the same values of $\gamma_{0} \phi$. Furthermore, the position of the maximum was found to be approximately 
equal to the critical volume fraction $\left(\Phi_{c}=\gamma_{c} \phi \approx 0.4\right)$ at which the oscillatory rheology indicates the onset of an elastic response [16]. The number of contacts was found to suddenly increase when comparing results for $\gamma_{0} \phi \geqslant \Phi_{c}$ to those for $\gamma_{0} \phi<\Phi_{c}$, but the number of contacts remained finite even at the smallest values of $\gamma_{0} \phi$ that were studied. Hence, the particle positions were irreversible over all conditions examined.

More certainty regarding this scaling, as well as the development of additional scaling principles, would require results spanning a larger set of concentrations and aspect ratios, but the range over which any scalings can be made or would be applicable are limited. Vorticity alignment occurs only over a narrow set of conditions which correspond to concentrated suspensions $\left(n L^{2} d \gtrsim 1\right)$ having an isotropic orientation distribution in the bulk. For these conditions, results given in Sec. IV A demonstrate that fibers adjacent to the confining walls are initially organized, at least loosely, and that oscillating can drive additional fiber organization and alignment with the vorticity direction. Creating suspensions with $n L^{2} d \gg 1$ requires a nematic orientation distribution, where the specific value of $n L^{2} d$ at which the transition to nematic ordering occurs is a decreasing function of the aspect ratio [31]. For these conditions, the fibers would be initially organized throughout the gap in addition to near the wall, and the dynamic changes caused by oscillating the suspension would likely differ significantly from the cases studied here.

\section{ACKNOWLEDGMENTS}

The assistance of Jessica Ofarrill with analyzing images was greatly appreciated. This work was supported by the National Science Foundation (Grants No. 1511787 and No.1362060). This material is also based upon research supported by the Chateaubriand Fellowship of the Office for Science \& Technology of the Embassy of France in the United States.

[1] B. J. Ackerson and P. N. Pusey, Shear-Induced Order in Suspensions of Hard Spheres, Phys. Rev. Lett. 61, 1033 (1988).

[2] B. J. Ackerson, Shear induced order and shear processing of model hard sphere suspensions, J. Rheol. 34, 553 (1990).

[3] M. D. Haw, W. C. K. Poon, and P. N. Pusey, Direct observation of oscillatory-shear-induced order in colloidal suspensions, Phys. Rev. E 57, 6859 (1998).

[4] T. Solomon and M. J. Solomon, Stacking fault structure in shear-induced colloidal crystallization, J. Chem. Phys. 124, 134905 (2006).

[5] T. H. Besseling, M. Hermes, A. Fortini, M. Dijkstra, A. Imhof, and A. van Blaaderen, Oscillatory shearinduced 3D crystalline order in colloidal hard-sphere fluids, Soft Matter 8, 6931 (2012).

[6] P. Panine, T. Narayanan, J. Vermant, and J. Mewis, Structure and rheology during shear-induced crystallization of a latex suspension, Phys. Rev. E 66, 022401 (2002).

[7] J. M. Bricker and J. E. Butler, Oscillatory shear of suspensions of noncolloidal particles, J. Rheol. 50, 711 (2006).

[8] Y. Lin, N. Phan-Thien, and B. C. Khoo, Short-term and long-term irreversibility in particle suspensions undergoing small and large amplitude oscillatory stress, J. Rheol. 57, 1325 (2013).

[9] J. M. Bricker and J. E. Butler, Correlation between stresses and microstructure in concentrated suspensions of non-Brownian spheres subject to unsteady shear flows, J. Rheol. 51, 735 (2007).

[10] D. J. Pine, J. P. Gollub, J. F. Brady, and A. M. Leshansky, Chaos and threshold for irreversibility in sheared suspensions, Nature (London) 438, 997 (2005).

[11] P. Pham, J. E. Butler, and B. Metzger, Origin of critical strain amplitude in periodically sheared suspensions, Phys. Rev. Fluids 1, 022201 (2016).

[12] J. M. McMullan and N. J. Wagner, Directed self-assembly of suspensions by large amplitude oscillatory shear flow, J. Rheol. 53, 575 (2009). 
[13] M. Joy, T. Muangnapoh, M. A. Snyder, and J. F. Gilchrist, Flow-induced alignment of (100) fcc thin film colloidal crystals, Soft Matter 11, 7092 (2015).

[14] M. H. Lash, M. V. Fedorchak, J. J. McCarthy, and S. R. Little, Scaling up self-assembly: Bottom-up approaches to macroscopic particle organization, Soft Matter 11, 5597 (2015).

[15] A. Franceschini, E. Filippidi, É. Guazzelli, and D. J. Pine, Transverse Alignment of Fibers in a Periodically Sheared Suspension: An Absorbing Phase Transition with a Slowly Varying Control Parameter, Phys. Rev. Lett. 107, 250603 (2011).

[16] A. Franceschini, E. Filippidi, É. Guazzelli, and D. J. Pine, Dynamics of non-Brownian fiber suspensions under periodic shear, Soft Matter 10, 6722 (2014).

[17] B. Snook, É. Guazzelli, and J. E. Butler, Vorticity alignment of rigid fibers in an oscillatory shear flow: Role of confinement, Phys. Fluids 24, 121702 (2012).

[18] O. L. Forgacs and S. G. Mason, Particle motions in sheared suspensions: IX. Spin and deformation of threadlike particles, J. Colloid Sci. 14, 457 (1959).

[19] R. G. Cox, The motion of long slender bodies in a viscous fluid Part 1. General theory, J. Fluid Mech. 44, 791 (1970).

[20] G. K. Batchelor, Slender-body theory for particles of arbitrary cross-section in Stokes flow, J. Fluid Mech. 44, 419 (1970).

[21] R. R. Sundararajakumar and D. L. Koch, Structure and properties of sheared fiber suspensions with mechanical contacts, J. Non-Newtonian Fluid Mech. 73, 205 (1997).

[22] J. Wu and C. K. Aidun, A numerical study of the effect of fibre stiffness on the rheology of sheared flexible fibre suspensions, J. Fluid Mech. 662, 123 (2010).

[23] J. E. Butler and B. Snook, Microstructural dynamics and rheology of suspensions of rigid fibers, Annu. Rev. Fluid Mech. 50, 299 (2018).

[24] F. P. Bretherton, The motion of rigid particles in a shear flow at low Reynolds number, J. Fluid Mech. 14, 284 (1962).

[25] B. J. Trevelyan and S. G. Mason, Particle motions in sheared suspensions. I. Rotations, J. Colloid Sci. 6, 354 (1951).

[26] S. G. Mason and R. St J. Manley, Particle motions in sheared suspensions: Orientations and interactions of rigid rods, Proc. R. Soc. London, Ser. A 238, 117 (1956).

[27] G. B. Jeffery, The motion of ellipsoidal particles immersed in a viscous fluid, Proc. R. Soc. London, Ser. A 102, 161 (1922).

[28] S. Gallier, E. Lemaire, F. Peters, and L. Lobry, Rheology of sheared suspensions of rough frictional particles, J. Fluid Mech. 757, 514 (2014).

[29] S. Gallier, E. Lemaire, L. Lobry, and F. Peters, Effect of confinement in wall-bounded non-colloidal suspensions, J. Fluid Mech. 799, 100 (2016).

[30] A. Newell, J. C. Shaw, and H. A. Simon, Empirical explorations of the logic theory machine: A case study in heuristic, in Papers Presented at the February 26-28, 1957, Western Joint Computer Conference: Techniques for Reliability, IRE-AIEE-ACM '57 (Western) (Association for Computing Machinery, New York, 1957), p. 218.

[31] S. R. Williams and A. P. Philipse, Random packings of spheres and spherocylinders simulated by mechanical contraction, Phys. Rev. E 67, 051301 (2003). 